\title{
Acute Lymphoblastic Leukemia in Adults - an Analysis of 51 Cases from a Tertiary Care Center in Pakistan
}

\author{
Sadia Sultan ${ }^{1 *}$, Syed Mohammed Irfan ${ }^{1}$, Saira Parveen ${ }^{1}$, Sanober Mustafa ${ }^{2}$
}

\begin{abstract}
Background: Acute lymphoblastic leukemia (ALL) is a malignant disease in which early lymphoid precursors proliferate and replace the normal hematopoiesis. It has distinctive clinical and biological features. In respect to adult ALL, available data from Pakistan are limited. Therefore we reviewed the demographical and clinicohematological profiles along with FAB stratification of adult patients with ALL presented at our hospital. Materials and Methods: In this cross sectional study, 51 adults ( $\geq 15$ years) patients with ALL were enrolled from January 2010 to December 2014. Results: The mean age was $23.8 \pm 12.9$ years with the median age of 18.0 years. The male to female ratio was $2: 1$. The major complaints were fever $(60.7 \%)$, generalized weakness $(47.0 \%)$, overt bleeding $(19.6 \%)$ and weight loss $(13.7 \%)$. Physical examination revealed lymphodenopathy as a predominant finding detected in $43.1 \%$ followed by splenomegaly and hepatomegaly in $23.5 \%$ and $21.5 \%$, respectively. The mean hemoglobin level was $9.0 \pm 2.75 \mathrm{~g} / \mathrm{dl}$ with a mean MCV of $82.2 \pm 15.4 \mathrm{fl}$, a mean total leukocyte count of $31.1 \pm 64.0 \times 10^{9} / \mathrm{l}$, a mean ANC of $2.1 \pm 3.0 \times 10^{9} / 1$ and a mean platelet count of $71.7 \pm 85.7 \times 10^{9} / 1$. According to FAB classification, $47.1 \%$ were $\mathrm{L} 1$ type, $45.1 \% \mathrm{~L} 2$ and $7.8 \% \mathrm{L3}$ variant. Conclusions: Clinico-pathological features appeared comparable to published data. Febrile illness associated with lymphodenopathy was the commonest presentation. FAB classification revealed a predominance of ALL-L1 variant in Pakistani adult patients with ALL.
\end{abstract}

Keywords: Acute lymphoblastic leukemia - adults - Pakistan

Asian Pac J Cancer Prev, 17 (4), 2307-2309

\section{Introduction}

Acute lymphoblastic leukemia (ALL) is a clonal disease characterized by malignant proliferation and accumulation of lymphoblast in the medullary cavity (Shaikh et al., 2014). Adults' acute lymphoblastic leukemia is a heterogeneous disease, due to the difference in disease biology and associated genetics abnormalities (Sabir et al., 2012). Acute lymphoblastic leukemia remains one of the most demanding and challenging adult malignancies, especially with respect to therapy (Pui et al., 2008). To date, there is no tumor registry maintained in Pakistan to keep a record regarding the prevalence and incidence of various malignancies including leukemia's.

The age adjusted overall incidence of ALL in the United States is 1.7/ 100,000 individuals (NCCN guidelines, 2015) with peaks between ages 2 years and 5 years and again after age 50 years (Jemal et al., 2006; Seiter., 2014). ALL represents $75 \%$ to $80 \%$ of acute leukemias among pediatric age group, making it the most common form of childhood leukemia; by contrast, ALL represents $\sim 20 \%$ of all leukemias amongst adults (NCCN guideline, 2015).

Most reports about etiological associations of ALL are conflicting and unclear (Jiang et al., 2013). The disease symptoms are predominantly caused by replacement of normal hemopoeitic marrow with leukemic cells, which causes an abrupt decline in normal peripheral blood counts. Clinical manifestations at presentation include constitutional symptoms (fevers, night sweats and weight loss), fatigue, easy bruising or bleeding, dyspnea and frequent infections. Bone, extremity and joint pain may be the only presenting symptoms (Greaves et al., 2006; Wartenberg et al., 2008).

Leukemia cells usually invade the peripheral blood very quickly. Eventually they can spread to other parts of the body, including the lymph nodes, liver, spleen, central nervous system and testes. This lymphoid deposits resulting in lymphodenopathy, hepatosplenomegaly, CNS manifestations and testicular enlargement in variable proportion of patients.

Currently there is limited data available regarding the epidemiological features of adults ALL in Pakistan, however, pediatric ALL are frequently reported from our part of world. The purpose of this study is to demonstrate demographical, FAB stratification, clinical and the hematological features of adults ALL patients who visited our tertiary care center from 2010 till the end of 2014. 


\section{Materials and Methods}

This descriptive cross sectional study was done at hematology department of Liaquat National Hospital, over a period of 5 years from January 2010 to December 2014.

All patients diagnosed as ALL who were $\geq 15$ years of age were included in the study. Patients with other lymphoid neoplasm were excluded. Patients with another associated malignancy or having relapsed/ refractory ALL were also excluded.

Based on this, a total of 51 subjects with newly diagnosed untreated de novo ALL were included in the analysis. The diagnosis of ALL was established according to the standard FAB criteria, and was based on bone marrow morphology and cytochemistry (Bennett et al., 1976).

Complete blood counts were determined by Automated Cell Dyne counter. Bone marrow samples were taken from posterior iliac crest through Jamshidi needle and were stained by Leishman's stain. Cytochemistry were carried out on each bone marrow smears including Sudan Black B (SBB), Periodic acid-Schiff (PAS) and Alphanaphthyl acetate esterase by commercially provided kits from Merck Diagnostic. Immunophenotyping was done in selected cases in patients with diagnostic uncertainty.

An ethical approval to conduct this analysis was granted by the institutional ethical review committee.

\section{Statistics analysis}

Data was assembled and analyzed using the Statistical Package for the Social Sciences version 22.0 (SPSS Inc, Chicago, IL, USA). The results were expressed as mean (SD) for quantitative variables and are presented as frequency \& percentages for qualitative variables.

\section{Results}

Out of 51 patients, 34 were males $(66.6 \%)$ and 17 were females $(33.3 \%)$ with male to female ratio of $2: 1$. Age ranged between 15 and 68 years with a mean age of $23.80 \pm 12.89$ years and median age of 18.0 years. Majority of patients $(80.3 \%)$ were under 30 years of age. Age stratification is shown in table-1.

According to FAB classification, 47.1\% had ALL-L1 type; $45.1 \%$ showed ALL-L2 type; while $7.8 \%$ having ALL-L3 variant.

The major complaints were fever in $31(60.7 \%)$ patients; generalized weakness in $24(47.0 \%)$ patients; bleeding in $10(19.6 \%)$ patients and weight loss in 7 (13.7\%) patients. The most common sites of bleeding were skin, nostrils and gastrointestinal tract.

Physical examination revealed lymphodenopathy as a predominant finding detected in $22(43.1 \%)$ patients followed by splenomegaly and hepatomegaly in 12 $(23.5 \%)$ and $11(21.5 \%)$ patients respectively.

The mean hemoglobin was $9.0 \pm 2.75 \mathrm{~g} / \mathrm{dl}$ with the mean MCV of $82.2 \pm 15.44 \mathrm{fl}$. The mean total leukocyte count of $31.06 \pm 63.99 \times 10^{9} / 1$; mean ANC of $2.1 \pm 3.0 \times 10^{9} / 1$ and the mean platelets count were $71.7 \pm 85.69 \times 10^{9} / 1$.

Anemia $(\mathrm{Hb}<10 \mathrm{gm} / \mathrm{dl})$ was noted in $33(64.7 \%)$ patients. Hyperleukocytosis (TLC count $>100 \times 10^{9} / 1$ ) was
Table 1.Age Stratification in Adult Patients with Acute Lymphoblastic Leukemia

\begin{tabular}{lcccc}
\hline Age groups & $\begin{array}{c}\text { Male } \\
\mathrm{n}=34\end{array}$ & $\begin{array}{c}\text { Female } \\
\mathrm{n}=17\end{array}$ & $\begin{array}{c}\text { Total } \\
\mathrm{n}=51\end{array}$ & $\begin{array}{c}\text { Percentage } \\
\%\end{array}$ \\
\hline $15-30$ & 27 & 14 & 41 & 80.3 \\
$31-50$ & 4 & 2 & 6 & 11.7 \\
$51-65$ & 1 & 1 & 2 & 3.9 \\
$65-68$ & 2 & 0 & 2 & 3.9 \\
\hline
\end{tabular}

seen in $4(7.8 \%)$ patients. Thrombocytopenia (platelets count $\left.<100 \times 10^{9} / 1\right)$ was detected in $41(80.3 \%)$ patients, while severe thrombocytopenia (platelets $<20 \times 10^{9} / 1$ ) was seen in $15(29.4 \%)$ patients.

\section{Discussion}

Acute lymphoblastic leukemia is characterized by monoclonal expansion of lymphoblasts, which encompass highly heterogeneous clinical entities. Disease progresses very rapidly and is fatal within weeks or months if left untreated. Though, major success has been achieved in the pediatric ALL, but results in adults ALL are still not influential.

French American British (FAB) classification for acute lymphoid leukemia had been widely accepted and applicable due to its ease in Pakistan. The present study has shown the existing spectrum of FAB classification in Pakistani patients with adults ALL. We determined ALL-L1 as the commonest type, followed by L2 and L3 respectively. Previously Hassan and recently Shahab had also determined similar findings in our population (Hassan et al., 1994; Shahab and Raziq., 2014).

ALL can occur in patients of any age and in general the overall incidence decline with the age (Shaikh et al., 2014). According to the National comprehensive cancer network (NCCN), 58.8\% of ALL patients are diagnosed at younger than 20 years of age. In contrast, $25.5 \%$ of cases are diagnosed at 45 years or older and only approximately $11 \%$ of patients are diagnosed at 65 years or older (NCCN guidelines., 2015). Similarly, the mean age of the patients in our study is 23.8 years. Previously published studies from Pakistan revealed variable age at disease presentation; as low 15.6 to as high as 33 years (Khalid et al., 1997; Shahab and Raziq., 2014).

Similar to us a large regional study reported by Malhotra et al from neighbor India, revealed the median age of ALL patients as 23 years at disease presentation (Malhotra et al., 2007). One Chinese study by Tong et al reported the mean age of 17.4 years at diagnosis (Tong et al., 2010). When compared with earlier study from Saudi Kingdom, findings are more or less similar with the median age of 31.5 years (Elyamany et al., 2014).

ALL is more frequent in men, with a male to female ratio of $\sim 2: 1$. In the present study male preponderance (ratio 2:1) was observed and it was consistent with that were reported in international and regional studies (Shahab and Raziq, 2014; Hayakawa et al., 2014; De França et al., 2014).

Generally, attenuation in hematopoiesis owing to clonal expansion of lymphoblast, subsequently lead to 
peripheral cytopenia with the clinical symptoms of pallor, easy fatigability, lethargy, bleeding manifestations and febrile illness. In the present study, majority of patients presented with fever $(60.7 \%)$ and generalized weakness (47\%). Similar presenting complaints were observed by prior Iranian and Pakistani studies, detected febrile illness in $61 \%$ and $63 \%$ respectively (Khalid et al., 1997; Mashhadi et al., 2012).

Hemorrhage (19.6\%) was also a common presenting feature in our series. Comparable findings have been reported in other studies on adult's ALL. When compared with earlier report, our results are in concurrence with a Bangladeshi study which reported fever and bleeding manifestation in $66 \%$ and $34 \%$ respectively (Islam et al., 2014).

Lymphadenopathy is not uncommon in acute lymphoblastic leukemia. Peripheral lymphodenopathy was found as a presenting symptom in $43.1 \%$ of our patients. One study from India reported comparatively higher prevalence $(61 \%)$ for lymphodenopathy (Malhotra et al., 2007). Subsequently, Islam also reported lymphodenopathy and hepatomegaly in $39 \%$ and $64 \%$ of patients respectively (Islam et al., 2014). However, enlarged lymph nodes are determined in $37 \%$ of patients in an earlier study from Pakistan (Khalid et al., 1997).

Among haemato-pathological findings $64.7 \%$ patients had $\mathrm{Hb}$ level $\leq 10 \mathrm{gm} / \mathrm{dl}, 80.3 \%$ patients had platelet count $\leq 100 \times 10^{9} / \mathrm{L}$ and $7.8 \%$ patients had WBC count $\geq 100 \times 10^{9} /$ L. Evaluations of hematological parameters in our cases showed predominance of anemia and thrombocytopenia. These findings are analogous with other studies from Oman and Bangladesh (Islam et al., 2014; Goud et al., 2015).

Amongst adult patients with acute leukemias, approximate 5 to $30 \%$ might present with symptoms of leukostasis and hyperleukocytosis (Asif and Hassan, 2013). However, hyperleukocytosis are much more pronounced in AML as compared with ALL (Asif and Hassan, 2013). In the present study $7.8 \%$ of patients showed hyperleukocytosis. An earlier regional study from India revealed $15.3 \%$ prevalence of hyperleukocytosis in their patients with ALL (Malhotra et al., 2007).

We acknowledge limitations of our study, including its retrospective nature and relatively small sample study. Another limitation was the unavailability of data concerning the treatment of our patients.

In conclusion, clinico-pathological features are appearing comparable to published data. Febrile illness associated with peripheral lymphodenopathy is the commonest presentation. FAB classification revealed predominance of ALL-L1 variant in Pakistani adult's patients with ALL.

\section{References}

Asif N, Hassan K (2013). Acute Myeloid Leukemia amongst Adults. J Islamabad Med Dental College, 2, 58-63.

Bennettt JM, Catovsky D, Daniel MT (1976). Proposals for the classification of the acute leukaemias. French-AmericanBritish (FAB) co-operative group. Br J Haematol, 33, 451-8.

De Franca Azevedo I, da Silva Junior RM, de Vasconcelos
AV, et al (2014). Frequency of p190 and p210 BCR-ABL rearrangements and survival in Brazilian adult patients with acute lymphoblastic leukemia. Rev Bras Hematol Hemoter, 36, 351-5.

Elyamany G, Awad M, Alsuhaibani O, et al (2014). FLT3 Internal Tandem Duplication and D835 Mutations in Patients with Acute Lymphoblastic Leukemia and its Clinical Significance. Mediterr J Hematol Infect Dis, 6, 2014038.

Greaves M (2006). Infection, immune responses and aetiology of childhood leukaemia. Nat Rev Cancer, 6, 193-203.

Goud TM,Al Salmani KK, Al Harasi SM, et al (2015). Importance of FISH combined with morphology, immunophenotype and cytogenetic analysis of childhood/adult acute lymphoblastic leukemia in omani patients. Asian Pac J Cancer Prev, 16, 7343-50.

Hassan K, Ikram N, Shah SH (1994). A morphological pattern of 234 cases of leukemias. J Pak Med Assoc, 44, 145-8.

Hayakawa F, Sakura T, Yujiri T, et al (2014). Markedly improved outcomes and acceptable toxicity in adolescents and young adults with acute lymphoblastic leukemia following treatment with a pediatric protocol: a phase II study by the Japan Adult Leukemia Study Group. Blood Cancer J, 17, 4-252.

Islam N, Rahman MM, Aziz MA, et al (2014). Clinical and haemato-pathological characteristics of adult acute lymphoblastic leukaemia. Mymensingh Med J, 23, 281-5.

Jemal A, Siegel R, Ward E, et al (2006). Cancer statistics.CA Cancer J Clin, 56, 106-30.

Jiang Y, Hou J, Zhang Q, et al (2013). The MTHFR C677T polymorphism and risk of acute lymphoblastic leukemia: an updated meta-analysis based on 37 case-control studies. Asian Pac J Cancer Prev, 14, 6357-62.

KhalidA,Zahid M,RehmanA, et al (1997).Clinicoepidemiological features of adult leukemias in Pakistan. J Pak Med Assoc, 47, 119-22.

Mashhadi MA, Koushyar MM, Mohammadi M (2012).Outcome of adult acute lymphoblastic leukemia in South East of iran (zahedan). Iran J Cancer Prev, 5, 130-7.

Malhotra P, Varma S, Varma N, et al (2007). Outcome of adult acute lymphoblastic leukemia with BFM protocol in a resource-constrained setting. Leuk Lymphoma, 48, 1173-8.

NCCN-National comprehensive cancer network, clinical practice guidelines in oncology, acute lymphoblastic leukemia, version 2015, MS2.

Pui CH, Robison L, Look AT (2008). Acute lymphoblastic leukemia. Lancet, 354, 166-178.

Seiter K (2014). Sarkodee-Adoo, C; Talavera, F; Sacher, RA; Besa, EC, ed. "Acute Lymphoblastic Leukemia”. Medscape Reference. WebMD. Retrieved 17 April 2014.

Sabir N, Iqbal Z, Aleem A, et al (2012). Prognostically significant fusion oncogenes in Pakistani patients with adult acute lymphoblastic leukemia and their association with disease biology and outcome. Asian Pac J Cancer Prev, 13, 3349-55.

Shaikh MS, Adil SN, Shaikh MU, Khurshid M (2014). Frequency of chromosomal abnormalities in Pakistani adults with acute lymphoblastic leukemia. Asian Pac J Cancer Prev, 15, 9495-8.

Shahab F, Raziq F (2014). Clinical presentations of acute leukemia. J Coll Physicians Surg Pak, 24, 472-6.

Tong H, Zhang J, Lu C, et al (2010). Immunophenotypic, cytogenetic and clinical features of 113 acute lymphoblastic leukaemia patients in China. Ann Acad Med Singapore, 39, 49-53.

Wartenberg D, Groves FD, Adelman AS (2008). Acute lymphoblastic leukemia: epidemiology and etiology. In: Estey EH, Faderl S, Kantarjian H, eds. Acute Leukemias. $1^{\text {st }}$ ed. Berlin, Germany: Springer; 2008, 77-93. 